\title{
Delayed Hem-o-Lok Clip Erosion Following Salvage Radical Prostatectomy
}

\author{
Philip G. Wong, PhD, Samay Jain, MD \\ University of Toledo College of Medicine, Toledo, OH, USA (all authors). \\ Department of Urology, University of Toledo Medical Center, Toledo, OH, USA (Dr. Jain).
}

\begin{abstract}
Introduction: Weck Hem-o-Lok (Teleflex Medical, Research Triangle Park, North Carolina) clips are frequently used to achieve hemostasis of the lateral prostatic pedicles during radical prostatectomy. Rarely, these clips can detach and migrate into the bladder wall, leading to postoperative urinary tract complications. Hem-o-Lok clip migration into the bladder is a rare complication of laparoscopic radical prostatectomy that has been reported within 1 year postsurgery.

Case Report: We report the case of a 61-year-old white man who presented with urinary stress incontinence, acute urinary retention, and a history of recurrent urinary tract infections more than 2 years after salvage robot-assisted laparoscopic radical prostatectomy for recurrent prostate cancer that was unsuccessfully treated with brachytherapy. As part of his diagnostic workup, cystoscopy demonstrated an open bladder neck with no evidence of contracture and the presence of a calcification near the vesicourethral anastomosis. Unroofing these calcifications via laser lithotripsy revealed two eroded Hem-o-Lok clips, which were subsequently removed from the bladder wall.
\end{abstract}

Conclusion: Salvage prostatectomy after radiotherapy as the primary treatment for prostate cancer may increase the window of time in which intravesical clip migration can occur because of delayed wound healing at the vesicourethral anastomosis. Furthermore, clip migration can present with signs of urinary tract dysfunction in the absence of bladder neck contracture and beyond the time frame initially expected.

Key Words: Prostatic neoplasms, Prostatectomy, Laparoscopy, Urinary incontinence, Postoperative complications.

Citation Wong PG, Jain S. Delayed Hem-o-Lok Clip Erosion Following Salvage Radical Prostatectomy. CRSLS e2014.001683. DOI: 10.4293/CRSLS.2014.001683.

Copyright (C) 2014 SLS This is an open-access article distributed under the terms of the Creative Commons Attribution-Noncommercial-ShareAlike 3.0 Unported license, which permits unrestricted noncommercial use, distribution, and reproduction in any medium, provided the original author and source are credited.

Address correspondence to: Samay Jain, MD, Department of Urology, University of Toledo, 3000 Arlington Ave - Mailstop 1091, Toledo, OH 43614 USA. Telephone (419) 383-6611, Fax: (419) 383-3785, E-mail: Samay.Jain@utoledo.edu

\section{INTRODUCTION}

Prostate cancer recurrence can occur after primary brachytherapy in $20 \%$ to $30 \%$ of cases, necessitating further intervention. $^{1}$ A slowly increasing prostate-specific antigen (PSA) level, with a large doubling time, after radiation therapy can indicate local treatment failure or prostate cancer recurrence. ${ }^{1}$ Local recurrence of prostate cancer after radiation therapy can be treated by several different modalities, including salvage radical prostatectomy. Although surgery is feasible, its complexity can be compounded by the previous radiation treatment. ${ }^{2}$ Radiation can result in fibrosis, damage to normal tissue, and compromised tissue planes, thus increasing the chances of significant complications during and after surgery. ${ }^{3}$ Increased risk of urinary tract infection (UTI), bladder neck contracture, urinary retention, urinary fistula, abscess, and rectal injury can occur more often as a result of salvage prostatectomy than nonsalvage procedures. ${ }^{4}$

Hem-o-Lok nonabsorbable polymer clips (Teleflex Medical, Research Triangle Park, North Carolina) represent an increasingly popular modality for blood vessel and structural ligation during laparoscopic and minimally invasive procedures, owing to their ease of application and effectiveness. ${ }^{5}$ These clips are used in a broad scope of general surgical and urologic procedures that include, but are not limited to, appendectomy, splenectomy, hysterectomy, lung lobectomy, cholecystectomy, and prostatectomy. ${ }^{5-7}$ Failure of Hem-o-Lok clips has resulted in intraoperative or postoperative bleeding (when they were used for blood vessel ligation) and bile leakage after laparoscopic 
cholecystectomy. ${ }^{5}$ The Food and Drug Administration has contraindicated the use of Hem-o-Lok clips in live-donor nephrectomies as a means to ligate the renal artery because of several reported hemorrhagic deaths subsequent to clip failure. ${ }^{8}$ In addition, cases of Hem-o-Lok clip migration into the first part of the duodenum ${ }^{9}$ and the common bile duct ${ }^{10}$ have been reported as a complication when the clips were used to ligate blood vessels and the cystic duct during laparoscopic cholecystectomy and common bile duct exploration.

Wide use of Hem-o-Lok clips in laparoscopic urology procedures, ${ }^{5}$ combined with a growing number of robotic-assisted laparoscopic radical prostatectomy (RALRP) procedures, has resulted in increases in procedural-specific complications. Several reports have described migration of the Hem-o-Lok clips, used for ligation of the vascular prostatic pedicle, through the vesicourethral anastomosis, leading to bladder neck contracture or intravesical calculus formation. ${ }^{11-18}$ However, clip migration is not isolated to the bladder neck: cases of clip erosion into the rectum ${ }^{19}$ and small bowel ${ }^{20}$ have also been reported. In this report, we describe a unique delayed presentation of a Hem-o-Lok clip erosion after salvage RALRP.

\section{CASE REPORT}

Our patient was a 61-year-old white man who presented to our clinic with a UTI and urinary retention 26 months after undergoing salvage RALRP. In July 2009, he was treated with brachytherapy for localized, Gleason $3+3$ adenocarcinoma involving $4 \%$ of the prostatic tissue. His PSA level at the time was $9.4 \mathrm{ng} / \mathrm{mL}$. After treatment, the patient's PSA level did not fall below $2 \mathrm{ng} / \mathrm{mL}$ and began to rise within 1 year of brachytherapy. Subsequently, a second needle biopsy was performed in June 2010, which revealed adenocarcinoma involving $11 \%$ of the prostate tissue. His workup was negative for metastasis and he underwent salvage RALRP in October 2010. Hem-o-Lok clips were used for ligation of the prostatic pedicles, and a running vesicourethral anastomosis was performed using a double-armed Biosynsuture (Covidien, Mansfield, Massachusetts). During the surgery, an extensive amount of radiation changes in the tissue surrounding the prostate was noted.

The patient's postoperative course was complicated by stress urinary incontinence and recurrent UTIs.

Two years after his salvage RALRP, the patient was again admitted with a UTI and urinary retention. At this point, a bladder neck contracture was suspected and, as a result, the patient underwent cystoscopic examination, which revealed a wide-open bladder neck and a calcification located at the 5-o'clock position at the vesicourethral anastomosis. Follow-up rigid cystoscopic examination with laser lithotripsy revealed two eroded and closed Hem-o-Lok clips that had calcified over time (Figure 1).

Since his procedure, the patient has not had any further UTIs and has been completely incontinent. He underwent placement of an artificial urinary sphincter approximately 6 weeks postprocedure and is now continent and has resumed normal activity. His PSA nadir is 0 and has remained this way since his salvage prostatectomy.

\section{DISCUSSION}

During radical prostatectomy procedures, it is important to limit the use of electrocautery for hemostasis because the heat generated can result in thermal injury to the surrounding structures, such as the neurovascular bundles, urethra, or rectum. ${ }^{21} \mathrm{Hem}-\mathrm{o}-\mathrm{Lok}$ clips are one athermal tool used to ligate and transect the vascular pedicle of the prostate. However, these clips have been associated with very specific adverse events, the most common being arterial bleeding as a result of clip dislodgment. ${ }^{11,21} \mathrm{~A}$ more rare complication associated with Hem-o-Lok use is migration of the clip into the bladder via the vesicourethral anastomosis. Although rare, erosion of the clip into the bladder can represent significant morbidity for the patient and usually requires subsequent procedures to correct it.

In previous published reports, $84.2 \%(16 / 19)$ of the patients presented within 1 year of their surgery and $63.2 \%$ $(12 / 19)$ also had a bladder neck contracture at the time of Hem-o-Lok clip erosion. ${ }^{11-18}$ Also unique to the present case, none of the patients in the previous reports had undergone a salvage prostatectomy, whether robotic, laparoscopic, or open.

Although the exact mechanism for clip erosion is not known,

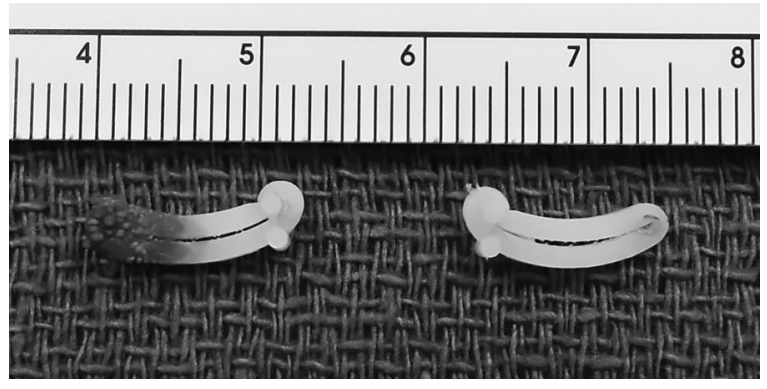

Figure 1. Hem-o-Lok clips removed from the bladder. 
previous case reports have hypothesized that vesicourethral anastomatic disruption may be a contributing factor. ${ }^{5}$ The concomitant finding of a bladder neck contracture with Hem-o-Lok clip erosion in many of the case reports ${ }^{11,12,15,16,18,22}$ supports this hypothesis because anastomotic leakage from disruption has been associated with bladder neck contracture in previous prostatectomy series. ${ }^{23}$

Our presented case differs from those previously reported because of its delayed presentation, absence of a bladder neck contracture, and the fact that it occurred after salvage, and not primary, RALRP. The normal sequence of wound healing can be compromised in tissue exposed to radiotherapy, potentially leading to delayed tissue repair. ${ }^{3,24}$ We suggest that in our current case, the irradiated vesicourethral anastomosis may have had a prolonged susceptibility to intravesical clip migration because of poor wound healing.

In our patient, the presence of recurrent UTIs may have also contributed to slowed anastomotic healing because a putative relationship between vesicourethral leakage after prostatectomy and UTIs has been described. ${ }^{25}$ Alternatively, the recurrent nature of the UTIs may suggest an etiology stemming from clip migration and radiation effects at the vesicourethral anastomosis. Ultimately, one or both of these factors impairing tissue repair at the vesicourethral anastomosis could have contributed to the extended time period before patient presentation. Interestingly, although radical prostatectomy, brachytherapy, and clip migration are individually associated with bladder neck contracture, ${ }^{11,26}$ the patient described here presented with his complications in the absence of a stricture.

In patients who are at risk for clip migration, especially those who have had previous radiation or surgery or recurrent infections, we suggest forgoing the use of permanent clips altogether. It may be prudent to use a degradable product or perform suture ligation of the area as an alternative to clip placement. However, these suggestions are based on theory alone, and a larger comparative study would need to be performed to validate these suggested technical changes.

To our knowledge, the case we present is the first reported incidence of Hem-o-Lok migration subsequent to a salvage prostatectomy. The initial presentation also represents the longest time interval between surgical intervention and discovery of intravesical clip migration, potentially because of previous radiotherapy and delayed healing at the vesicourethral anastomosis. The patient also presented uniquely with recurrent UTIs and lack of a bladder neck contracture. These findings em- phasize the importance of careful long-term monitoring for broad urinary tract symptoms after the use of Hemo-Lok clips in salvage radical prostatectomy. Complications of clip migration may present as symptoms other than bladder neck contracture, and beyond the time frame initially expected.

\section{References:}

1. Rukstalis DB. Treatment options after failure of radiation therapy-a review. Rev Urol. 200;4(Suppl 2):S12-S17.

2. Rocco B, Cozzi G, Spinelli MG, et al. Current status of salvage robot-assisted laparoscopic prostatectomy for radiorecurrent prostate cancer. Curr Urol Rep. 2012;13(3):195-201.

3. Stone HB, Coleman CN, Anscher MS, McBride WH. Effects of radiation on normal tissue: consequences and mechanisms. Lancet Oncol. 2003;4(9):529-536.

4. Gotto GT, Yunis LH, Vora K, Eastham JA, Scardino PT, Rabbani F. Impact of prior prostate radiation on complications after radical prostatectomy. J Urol. 2010;184(1):136-142.

5. Aminian A, Khorgami Z. Hem-O-Lok Clip is safe in minimally invasive general surgery: a single-center experience and review of data from Food and Drug Administration. Journal of Minimally Invasive Surgical Sciences. 2012;1(2).

6. Hue CS, Kim JS, Kim KH, Nam SH, Kim KW. The usefulness and safety of Hem-o-lok clips for the closure of appendicular stump during laparoscopic appendectomy. J Korean Surg Soc. 2013;84(1):27-32.

7. Delibegovic S, Iljazovic E, Katica M, Koluh A. Tissue reaction to absorbable endoloop, nonabsorbable titanium staples, and polymer Hem-o-lok clip after laparoscopic appendectomy. JSLS 2011;15(1):70-76.

8. Friedman AL, Peters TG, Ratner LE. Regulatory failure contributing to deaths of live kidney donors. Am J Transplant. 2012;12(4):829-834.

9. Seyyedmajidi M, Hosseini SA, Hajiebrahimi S, Vafaeimanesh $\mathrm{J}$. Hem-o-Lok clip in the first part of duodenum after laparoscopic cholecystectomy. Case Rep Gastrointest Med. 2013;251634.

10. Liu Y, Ji B, Wang Y, Wang G. Hem-o-lok clip found in common bile duct after laparoscopic cholecystectomy and common bile duct exploration: a clinical analysis of 8 cases. Int J Med Sci. 2012;9(3):225-227.

11. Blumenthal KB, Sutherland DE, Wagner KR, Frazier HA, Engel JD. Bladder neck contractures related to the use of Hemo-lok clips in robot-assisted laparoscopic radical prostatectomy. Urology. 2008;72(1):158-161.

12. Tunnard GJ, Biyani CS. An unusual complication of a Hemo-Lok clip following laparoscopic radical prostatectomy. J Laparoendosc Adv Surg Tech A. 2009;19(5):649-651. 
13. Tugcu V, Polat H, Ozbay B, Eren GA, Tasci AI. Stone formation from intravesical Hem-o-lok clip migration after laparoscopic radical prostatectomy. J Endourol. 2009;23(7): 1111-1113.

14. Banks EB, Ramani A, Monga M. Intravesical Weck clip migration after laparoscopic radical prostatectomy. Urology. 2008; 71(2):351, e353-354.

15. Moser RL, Narepalem N. Erosion of Hem-o-Lok clips at the bladder neck after robot-assisted radical prostatectomy. J Endourol. 2009;23(6):949-951.

16. Shin YS, Doo AR, Cha JS, Kim MK, Jeong YB, Kim HJ. Floating Hem-o-Lok clips in the bladder without stone formation after robot-assisted laparoscopic radical prostatectomy. Korean J Urol. 2012;53(1):60-62.

17. Mora ER, Gali OB, Garin JA, Arango O. Intravesical migration and spontaneous expulsion of a Hem-o-lok polymer ligating clip after laparoscopic radical prostatectomy. Urology. 2010; 75(6):1317.

18. Ganpule A, Dhawan DR, Desai MR. Hem-o-Lok clip eroding into the urethra following laparoscopic radical prostatectomy: a case report and review of literature. Indian J Urol. 2010;26(4): $580-581$.

19. Wu SD, Rios RR, Meeks JJ, Nadler RB. Rectal Hem-o-Lok clip migration after robot-assisted laparoscopic radical prostatectomy. Can J Urol. 2009;16(6):4939-4940.
20. Ghani KR, Hurwitz M, Menon M. Hem-o-lok clip causing small bowel obstruction after robot-assisted radical prostatectomy. Int J Urol. 2012;19(10):962-963.

21. Ahlering TE, Skarecky D, Borin J. Impact of cautery versus cautery-free preservation of neurovascular bundles on early return of potency. J Endourol. 2006;20(8):586-589.

22. Yi JS, Kwak C, Kim HH, Ku JH. Surgical clip-related complications after radical prostatectomy. Korean J Urol. 2010;51(10): 683-687.

23. Webb DR, Sethi K, Gee K. An analysis of the causes of bladder neck contracture after open and robot-assisted laparoscopic radical prostatectomy. BJU Int. 2009;103(7):957-963.

24. Haubner F, Ohmann E, Pohl F, Strutz J, Gassner HG. Wound healing after radiation therapy: review of the literature. Radiat Oncol. 2012;7:162.

25. Gnanapragasam VJ, Baker P, Naisby GP, Chadwick D. Identification and validation of risk factors for vesicourethral leaks following radical retropubic prostatectomy. Int J Urol. 2005; 12(11):948-952.

26. Elliott SP, Meng MV, Elkin EP, et al. Incidence of urethral stricture after primary treatment for prostate cancer: data From CaPSURE. J Urol. 2007;178(2):529-534, discussion 534. 